\title{
RELATIONAL SCHEMAS TO INVESTIGATE THE PROCESS OF LEADERSHIP EMERGENCE
}

\author{
CÉCILE EMERY \\ GIANLUCA CARNABUCI \\ DAVID BRINBERG \\ INTRODUCTION
}

The present paper develops new theory and evidence explaining how social structures emerge from a process of "social cognition," that is, how through situated action and interaction individuals define and stabilize their as well as others' position in a social structure (Foti, Knee, $\&$ Backert, 2008). We focus in particular on the emergence of leadership structures within informal social groups, examining how social cognition allows the leadership choices of individual group members to become progressively coordinated at the group level (Kilduff et al., 2008; Ridgeway, 2006). Our theoretical arguments draw from relational schema theory (Jannick \& Larrick, 2005), which posits that to be eligible for participation in any social group, individuals must be able to infer the relational pattern in which other group members are involved, and must define their own relational strategy in response (Fiske, 1992; Stryker \& Vryan, 2003; Jannick \& Larrick, 2005; Ridgeway, 2006). Relational schema theory argues that individuals reduce the uncertainty inherent in this process by relying on simplified and abstract cognitive representations of appropriate and desirable relational patterns (Baldwin, 1992). For example, past research has shown that people map the structure of friendship relations within a group by employing abstract cognitive representations in which these relations are expected to be "reciprocal" (if Mary is Jane's friend, then Jane ought to be Mary's friend) and "transitive" (if Mary and Jane are Rebecca's friends, then they ought to be each other's friends) (Jannick \& Larrick, 2005).

In the present paper, we investigate how relational schemas help group members to arrive at coordinated leadership choices, thereby creating the precondition for coherent leadership structures to emerge at the group level. As the aggregate pattern of interpersonal leadership relations within any but very small groups tends to be highly complex, forming accurate cognitive representations of the whole network of leadership relations is very difficult (Krackhardt, 1990; Krackhardt \& Kilduff, 1999). Based on prior research, we suggest that individuals reduce the complexity of this coordination problem in two related ways. First, they focus their attention on the local social structure immediately around them, rather than on the global leadership structure emerging at the level of the whole group (Martin, 2010). Second, they adopt simplified relational schemas whereby leadership relations are assumed to be "hierarchical" (Fiske, 1991, 1992; Haslam \& Fiske, 1999), establishing an inherently asymmetric social relation between a leader and his or her followers.

We develop a research design comprising two related studies to empirically test our hypotheses concerning the role of relational schemas in leadership emergence. The objective of Study 1 is to investigate, within two naturally occurring social groups, whether the evolution of individuals' leadership choices is driven by the hypothesized hierarchical relational schemas. To carry out such investigation, we follow prior studies and model leadership structure as a network 
of inter-personal leadership choices (Carson, Tesluk, \& Marrone, 2007; Mehra, Smith, Dixon, \& Robertson, 2006). By simultaneously capturing leadership choices at the individual, dyadic, and group level, leadership networks have been argued to provide a realistic yet analytically tractable conceptualization of leadership structures (Day \& Harrison, 2007; Livi, Kenny, Albright, \& Pierro, 2008; Yammarino, Dionne, Chun, \& Dansereau, 2005). Furthermore, while leadership networks have thus far been analyzed from a static perspective (Carson et al., 2007; Mehra et al., 2006), we adopt recently developed network models allowing us to examine how the leadership choices of individual group members co-evolve over time (Snijders, Steglich, \& van de Bunt, 2010). Study 2, on the other hand, is an experiment designed to test whether the identified relational schemas reflect people's mental representations of leadership structures in general, i.e., outside the specific social context in which the group operates. To this latter end, we sample a group of individuals that did not participate in Study 1, and create vignettes to assess whether the hypothesized relational schemas guide people's cognitive representations of leadership structure (DeSoto \& Kuethe, 1959).

\section{THEORY \& HYPOTHESES}

Social structure "is generated in everyday, situated interaction" (Howard, 1994: 218). However, making sense of new or different social situations does not require people to reconstruct social reality every time anew. Rather, people routinely represent, interpret, and act upon the social structure in which they are embedded by means of a relatively limited range of abstract and transposable cognitive schemas (Segal, 1988). Relational schemas are "cognitive structures representing regularities in patterns of interpersonal relatedness" (Baldwin, 1992: 461), which provide generalized representations of both self (Cervone \& Pervin, 2008) and other (Horowitz, 1988) and help individuals make sense of complex and incomplete social information (Janick \& Larrick, 2005; Ridgeway, 2006). Relational schemas provide the cognitive structure through which social structures are represented and encoded within people's mind, as well as enacted in actual social interactions in everyday life (Kilduff et al., 2008).

Building on this view, we propose that relational schemas provide a crucial coordination device through which leadership structures can emerge in groups. Relational schemas induce individuals to dynamically adjust their relational strategy "to be congruent with their schematic expectations concerning how relationships... should be structured" (Balkundi \& Kilduff, 2005: 944). Our contention is that in their attempt to make sense of leadership relations, individuals employ a limited set of default "hierarchical" schemas, helping them simplify, disambiguate, and fill in the blanks in their social information (Janick \& Larrick, 2005). DeRue and Ashford (2010: 627 ) argue that "leader and follower identities are... socially constructed and inherently related (e.g., granting one person a leader identity frequently instantiates a follower identity for others)." By cognitively representing leadership relations as asymmetric positions along a ranking order (Fiske, 1991, 1992; Haslam \& Fiske, 1999; Martin, 2010), we suggest that hierarchical relational schemas guide group members as they ascribe "leader" and "follower" identities to both themselves (Kassin, Fein, \& Markus, 2008) and their significant others (Martin, 2010).

\section{Dyadic Level: “Asymmetry” Schema}

A key component of relational schemas pertains to the cognitive representation of self in relation to other (Baldwin, 1992). In friendship relations, for example, individuals expect the 
self-other relation to be strictly symmetric. Our contention, conversely, is that people cognitively represent leadership relations as strictly asymmetric (Fernandez, 1991). Leadership is defined as a relation in which an individual is accepted as a leader by another who, either explicitly or implicitly, accepts to follow (Kouzes \& Posner, 2004). By recognizing someone else as a leader, the follower confers him or her both deference and influence. As such, leadership relations distinguish between non-equals (Brandts, Cooper, \& Fatas, 2007), and they are governed by rules and symbolic rituals involving precedence, hierarchy, status (Haslam, 1994). Hence, leaderfollower identities mutually constitute each other on the basis of their strictly asymmetric relation (Morgeson, DeRue, \& Karam, 2010).

This asymmetry provides an important coordination device influencing the evolution of leadership relations within a social group, because conceiving of oneself as somebody else's leader implies that the follower ought not be granted a similar leader's position. For example, if Mary conceives of herself as being Jane's leader, she is unlikely to grant Jane a leadership identity. Similarly, if Jane accepts Mary as a leader, she will expect Mary not to regard her as a leader. Through the course of actual social interaction, however, people may be confronted with behaviors and information inconsistent with the leadership structures implied by the asymmetry relational schema. For example, it is possible that although Jane regards Mary as a leader, Mary feels the same for Jane. Our argument is that when such breaches in the asymmetry principle occur, they induce individuals to adjust their leadership choices so that the experienced inconsistency is reduced over time. For example, learning that Mary regards her as a leader will likely increase the probability that Jane ceases to think of Mary as her leader. The argument that leadership choices are driven by an asymmetry relational schema leads to the following hypothesis.

Hypothesis 1: The probability that a group member will come to accept another as a leader declines if the latter accepts the former as a leader.

\section{Triadic Level: "Transitivity" and "A-Cyclicity" Schemas}

In addition to providing cognitive representations of self in relation to other, relational schemas encode knowledge about the expected structure of other-other relations (Crockett, 1982). For example, previous research has shown that people regard friendship relations as inherently transitive. If Mary is tied by a strong friendship relation to both Jane and Rebecca, then Mary expects Jane and Rebecca to be good friends too (Gollob, 1974).

We expect the transitivity rule to be encoded in leadership relational schemas as well. In the context of leadership relations, transitivity implies that if Mary accepts Jane to be her leader, and if Jane accepts Rebecca to be her leader, then it is likely that Mary will be induced to accept Rebecca as a leader. This argument is based on the premise that by recognizing Rebecca as a leader, Jane confers her both deference and influence (Brandts, Cooper, \& Fatas, 2007). Learning that Jane perceives Rebecca as a leader is therefore likely to impact Mary's view of Rebecca in two ways. On the one hand, Mary may be induced to consider choosing Rebecca as a leader simply because of the respect she holds for Jane's opinions and judgment. On the other hand, Mary may feel normatively pressured to confer Rebecca a leadership identity because not doing so could be perceived as a challenge towards Jane, thereby potentially destabilizing their leaderfollower relation. For both reasons, learning that Jane accepts Rebecca as a leader increases the probability that Mary will come to accept Rebecca as a leader too. 
The argument that leadership perceptions are driven by a transitivity relational schema leads to the following hypothesis.

Hypothesis 2: The probability that group member $i$, who accepts $j$ as a leader, will accept

$h$ as a leader increases if $j$ accepts $h$ as a leader.

Let us consider one more time a situation in which Mary perceives Jane as a leader, who in her turn perceives Rebecca as a leader. In addition to inducing Mary to perceive Rebecca as a leader (our second hypothesis), we expect this relational pattern to reduce the probability that Rebecca will come to accept Mary as a leader. This argument rests on the view that leadership choices are driven by a third formal property encoded in leadership relational schemas, which we will call $a$-cyclicity. The a-cyclicity principle may be derived as a logical extension of the asymmetry schema. If the asymmetry schema implies that Jane ought not to accept Mary as a leader, this same implication should be valid for Jane's leader, Rebecca. For example, suppose that over the course of her social interaction within the group Rebecca learns that Mary, whom she had chosen to accept as a leader, accepts Jane as a leader. Because Rebecca is Jane's leader, this discovery would reveal an inconsistency between the actual leadership structure that is developing around Rebecca and her own leadership choices. In order to reduce such inconsistency, Rebecca will likely reconsider her choice to accept Mary as a leader. Arguing that leadership choices are driven by an a-cyclicity relational schema leads to the following hypothesis.

Hypothesis 3: The probability that $i$ will accept $j$ as a leader decreases if $j$ accepts $h$ as a leader, who in turn accepts $i$ as a leader.

\section{Group Level: Centrality Schema}

Past research has argued that "an emerging leader who is perceived to be popular may benefit from a bandwagon effect: people may want to associate with someone perceived to be a rising star" (Balkundi \& Kilduff, 2006: 947). According to this view, Mary is more likely to accept Jane as a leader if she knows that many other group members do so. Conversely, discovering that fewer members of the group regard Jane as a leader increases the probability that Mary will cease to see her as a leader. We suggest that the bandwagon effect is triggered by a hierarchical relational schema whereby leaders are encoded as inherently more "central" than non-leaders within the group (Janicik \& Larrick, 2005: 361). Thus, finding out that Jane is regarded as a leader by a large share of group members provides Mary with a social cue that Jane corresponds to a leader identity. Arguing that leadership choices are driven by a centrality relational schema leads to the following hypothesis:

Hypothesis 4: The probability that $i$ will accept $j$ as a leader increases with the number of other group members that accept $j$ as a leader.

The centrality schema underlying the bandwagon effect yields a second testable implication. In addition to affecting Mary's cognitive representation of Jane's leadership position, if Jane is accepted as a leader by many group members she is more likely over time to conceive of herself as a leader, which in turn implies that she is less likely to regard other group members as leaders (Morgeson et al., 2010). This argument leads to the following hypothesis.

Hypothesis 5: The probability that $i$ will accept other group members as leaders decreases with the number of group members accepting $i$ as a leader.

\section{RESEARCH METHODS}




\section{Study 1: A dynamic analysis of leadership networks to identify the formal properties that define hierarchical local structures}

The objective of Study 1 is to investigate whether in a naturally occurring informal group, the evolution of individuals' leadership choices is driven by the leadership relational schemas hypothesized above. To carry out such investigation, we follow the evolving pattern of leadership relations among a group of University exchange students. Based on the model of shared leadership (Pearce, Conger, \& Locke, 2007), we represent the leadership structure emerging within this group as a network of leadership choices (Carson et al., 2007; Mehra et al., 2006) and track its evolution at subsequent points in time. Adopting recent models for the statistical analysis of longitudinal network data (e.g., Snijders, Steglich, \& van de Bunt, 2010), we then examine how the leadership choices of individual group members evolve over time. Corroborating our hypotheses, we find support for all our hypothesized relational schemas. This result indicates that people continuously adjust their leadership choices by comparing the actual leadership structure emerging around them with the default relational schemas associated to leadership, allowing a coherent leadership structure to emerge at the group level.

\section{Study 2: Assessing the relational schemas encoded in people's abstract representation of leadership relations}

Study 2 is an experiment designed to directly test whether the hypothesized relational schemas reflect people's mental representations of leadership structures in general. Following a long methodological tradition in cognitive psychology (DeSoto \& Kuethe, 1959), subjects are asked to provide subjective probability assessments that certain relational patterns could occur. Results from Study 2 confirm that the hypothesized relational schemas encode knowledge through which people cognitively represent leadership relations in general. The results of Study 2 indicate that the identified relational schemas are an important cognitive factor driving and coordinating people's leadership choices.

\section{DISCUSSION AND CONCLUSIONS}

The current study investigated how relational schemas help group members to arrive at coordinated leadership choices, thereby creating the precondition for coherent leadership structures to emerge. Following previous studies on social cognition (e.g., Jannick and Larrick 2005; Martin, 2010), our analytical strategy was to examine the formal properties defining hierarchical local structures to identify the relational schemas encoded in people's abstract representation of leadership relations. By encoding a set of default expectations about what leadership relations are and how they should be structured, we hypothesized such schemas to drive the formation and dissolution of leadership relations within social groups. Our argument was not that relational schemas directly cause the leadership choices of group members. Rather, we posited that individuals employ relational schemas to evaluate whether their leadership choices are appropriate, given the leadership structure that is emerging around them. Through social interaction within the group, people learn that their choice to (not) accept somebody as a leader is inconsistent with the abstract relational pattern encoded in a relational schema, they will be induced to reduce such inconsistency by reconsidering that leadership relation. 
Using an innovative multi-method research design, the paper contributes to the extant literature in three main ways. First, it advances leadership research by revealing that leadership emergence is structurally embedded in a process of social cognition, with leadership choices of individual group members evolving in an interdependent and coordinated fashion. The social construction of leadership suggests that as individual perceptual processes are embedded in the structure of the broader group, individual leadership choices are fundamentally interdependent. This argument stands in stark contrast with so-called "great man" theories of leadership emergence, which emphasize that leadership processes are primarily affected by characteristics of individuals. Departing from this latter line of inquiry, we see the present study as a first step towards understanding how individual perceptual processes are entrenched in the social structure of the group (Balkundi \& Kilduff, 2006; Lord \& Emrich, 2001).

We also extend relational schema theory by identifying a number of relational schemas that systematically affect people's representation of a fundamental aspect of social relations: leadership. While salient relational schemas have been identified with regard to people's representation of friendship (e.g., Krackhardt \& Kilduff, 1999) and influence (e.g., DeSoto, 1960), we focused on the role of relational schemas in the cognition of leadership relations. By doing so, our study extends the "empirical content" (Lakatos, 1978) of relational schema theory. Taken together, our findings illustrate the tendency for people to see leadership as hierarchical ordering. In people's mind, emergent leadership is perceived as inherently asymmetric and hierarchical - a principle that appears to be crucial in the social construction of both self and others' identity (DeRue \& Ashford, 2010).

Finally, by incorporating the explanatory logic of relational schema theory within the context of (leadership) network evolution, we further our understanding of how micro-cognitive mechanisms shape the emergence of social structure through a process of social cognition (Baldwin, 1992; Howard, 1994). We show that a limited set of relational schemas guide people's leadership choices, helping them to align and coordinate their individual leadership perceptions to the broader leadership structure emerging within the group. This finding is particularly important in that it suggests that for a coherent leadership structure to emerge at the group level, it is enough for people to attend to social cues coming from the local social structure immediately surrounding them. By substantiating and articulating the view that an orderly social structure may emerge from dynamics of social cognition involving socially proximate and rational individuals, the present study represents an attempt at reconciling structuralist and cognitive views of social order.

\section{REFERENCES AVAILABLE FROM THE AUTHORS}

